\title{
C17orf37 is a differentially expressed gene in brain metastatic human breast cancer.
}

Shahan Mamoor, $\mathrm{MS}^{1}$

${ }^{1}$ shahanmamoor@gmail.com

East Islip, NY USA

Metastasis to the brain is a clinical problem in patients with breast cancer ${ }^{1-3}$. We mined published microarray data ${ }^{4,5}$ to compare primary and metastatic tumor transcriptomes for the discovery of genes associated with brain metastasis in humans with metastatic breast cancer. We found that open reading frame 37 on chromosome 17, also known as the migration and invasion enhancer 1 (MIEN1), encoded by C17orf37, was among the genes whose expression was most different in the brain metastases of patients with metastatic breast cancer as compared to primary tumors of the breast. C17orf37 mRNA was present at increased quantities in brain metastatic tissues as compared to primary tumors of the breast. Modulation of $\mathrm{C} 17$ orf37 expression may be relevant to the biology by which tumor cells metastasize from the breast to the brain in humans with metastatic breast cancer.

$\underline{\text { Keywords: }}$ breast cancer, metastasis, brain metastases, central nervous system metastases, C17orf37, open reading frame 37 on chromosome 37, MIEN1, systems biology of breast cancer, targeted therapeutics in breast cancer. 
One report described a $34 \%$ incidence of central nervous system metastases in patients compared administration of either neratinib or trastuzumab in conjunction with paclitaxel demonstrated that in a randomized, controlled setting, in breast cancer patients treated with neratinib, not only was the incidence of central nervous system recurrence significantly lower, the time to central nervous system metastasis was significantly delayed as compared to patients administered trastuzumab ${ }^{6}$. The alarmingly high rate of central nervous system metastasis described, as well as data, both anecdotal ${ }^{2}$ and from a randomized, controlled setting ${ }^{6}$ illustrating that treatment with trastuzumab may be associated with these events demands an enhanced understanding of the transcriptional makeup of brain metastatic tissues to support identification of therapeutic targets, whether they are treatment related or not. We performed a global comparative analysis of primary and metastatic tumors in patients with brain metastatic breast cancer ${ }^{4,5}$. We discovered differential and increased expression of the gene encoding the open reading frame 37 on chromosome 17, C17orf37, in brain metastatic tissues of patients with metastatic breast cancer.

\section{$\underline{\text { Methods }}$}

We used datasets GSE $10893^{4}$ and GSE $42568^{5}$ for this global differential gene expression analysis of brain metastatic breast cancer in conjunction with GEO2R. GSE10893 was generated using Agilent-011521 Human 1A Microarray G4110A technology with $n=11$ primary breast tumors and $n=3$ brain metastases from patients with breast cancer; analysis was performed using platform GPL885. GSE42568 was generated using Affymetrix Human Genome U133 Plus 2.0 array technology with $n=17$ normal breast tissue biopsies and $n=104$ primary breast tumor biopsies from patients with breast cancer; analysis was performed using platform GPL570. The Benjamini and Hochberg method of $p$-value adjustment was used for ranking of differential expression but raw $p$-values were used to assess statistical significance of global differential expression. Log-transformation of data was auto-detected, and the NCBI generated category of platform annotation was used. A statistical test was performed to evaluate whether $\mathrm{C} 17$ orf 37 gene expression was significantly different between primary tumors of the breast and brain metastases in humans with breast cancer using a two-tailed t-test.

\section{$\underline{\text { Results }}$}

We performed global comparative transcriptome analysis of metastatic and primary tumor tissues of patients with metastatic breast cancer using published microarray data ${ }^{4,5}$ to describe the transcriptional landscape of brain metastasis in human breast cancer in an unbiased fashion and for the discovery of novel therapeutic targets.

\section{C17orf37 is differentially expressed in the brain metastases of patients with brain metastatic breast cancer.}

Through blind, systems-level analysis of published microarray data ${ }^{4}$, we identified the open reading frame 37 on chromosome 17, encoded by $\mathrm{C} 17$ orf37, also known as the migration and invasion enhancer MIEN1, MGC14832, C35, RDX12, XTP4, and Orb3, as a differentially expressed gene in the breast metastatic tissues of humans with breast cancer (Table 1). When sorting each of the genes expressed in brain metastases based on significance of difference as 
compared to primary tumors of the breast in patients with breast cancer, C17orf37 ranked 29 out of 17418 total transcripts (Chart 1), equating to $99.8 \%$ differential expression. Differential expression of $\mathrm{C} 17$ orf37 in the brain metastases of patients with metastatic breast cancer was statistically significant (Chart $1 ; p=3.76 \mathrm{E}-05)$.

To validate transcriptome-wide differential expression of C17orf37 in human breast cancer, we queried a second microarray datase ${ }^{5}$, here comparing normal breast tissues and primary tumors of the breast. Again, we identified C17orf37 as a differentially expressed gene in the brain metastatic tissues of patients with breast cancer (Chart 2). When sorting each of the genes expressed in brain metastases based on significance of difference as compared to normal breast tissues, C17orf37 ranked 19492 out of 54675 total transcripts (Chart 2), equating to 64.3\% differential expression. Differential expression of $\mathrm{C} 17$ orf37 in the primary tumors of patients with breast cancer approached the level of statistical significance (Chart 2; $p=3.37 \mathrm{E}-02$ ). Thus, differential expression of C17orf37, transcriptome-wide, in the tumor tissues of women with breast cancer was conserved across two independent microarray datasets, both in primary and metastatic tumor tissues.

\section{C17orf37 is expressed at higher levels in the brain metastases of patients with metastatic breast cancer.}

We obtained exact mRNA expression levels for C17orf37, in primary tumors of the breast and in brain metastasis of patients with brain metastatic breast cancer to determine direction and statistical significance of change in $\mathrm{C} 17$ orf37 expression in brain metastatic tissues. C17orf37 was expressed at higher levels in the brain metastases of patients with breast cancer as compared to primary tumors of the breast, and this difference was statistically significant (Figure 1; $p=0.000136)$.

Thus, by mining published microarray data ${ }^{4,5}$ in an unbiased fashion, we identified ATP-binding cassette transporter sub-family $\mathrm{C}$ member 8, encoded by $\mathrm{C} 17$ orf37, as among the genes whose expression was most different, transcriptome-wide, in the brain metastases and primary tumors of patients with breast cancer; we observed significantly increased expression of C17orf37 in brain metastases as compared to primary tumors of the breast.

\section{Discussion}

We provided evidence here that open reading frame 37 on chromosome 37 , encoded by C17orf37, is among the genes whose expression is most different in the brain metastases of patients with brain metastatic breast cancer, and that C17orf $37 \mathrm{mRNA}$ is present at significantly increased quantities in brain metastatic tissues as compared to primary tumors of the breast. Evaluation of the effects of genetic depletion of C17orf37 in mouse models of metastatic breast cancer on metastasis to the central nervous system is merited. Modulation of C17orf37 expression may be relevant to the processes by which breast cancer cells exit the breast, enter the vasculature and/or lymphatics, reside in the lymph nodes, evade immune clearance, breach the blood-brain barrier and colonize the brain. 


\section{References}

1. Lin, N.U., Amiri-Kordestani, L., Palmieri, D., Liewehr, D.J. and Steeg, P.S., 2013. CNS metastases in breast cancer: old challenge, new frontiers.

2. Bendell, J.C., Domchek, S.M., Burstein, H.J., Harris, L., Younger, J., Kuter, I., Bunnell, C., Rue, M., Gelman, R. and Winer, E., 2003. Central nervous system metastases in women who receive trastuzumab-based therapy for metastatic breast carcinoma. Cancer, 97(12), pp.2972-2977.

3. Tsukada, Y., Fouad, A., Pickren, J.W. and Lane, W.W., 1983. Central nervous system metastasis from breast carcinoma autopsy study. Cancer, 52(12), pp.2349-2354.

4. Weigman, V.J., Chao, H.H., Shabalin, A.A., He, X., Parker, J.S., Nordgard, S.H., Grushko, T., Huo, D., Nwachukwu, C., Nobel, A. and Kristensen, V.N., 2012. Basal-like Breast cancer DNA copy number losses identify genes involved in genomic instability, response to therapy, and patient survival. Breast cancer research and treatment, 133(3), pp.865-880.

5. Clarke, C., Madden, S.F., Doolan, P., Aherne, S.T., Joyce, H., O’driscoll, L., Gallagher, W.M., Hennessy, B.T., Moriarty, M., Crown, J. and Kennedy, S., 2013. Correlating transcriptional networks to breast cancer survival: a large-scale coexpression analysis. Carcinogenesis, 34(10), pp.2300-2308.

6. Awada, A., Colomer, R., Inoue, K., Bondarenko, I., Badwe, R.A., Demetriou, G., Lee, S.C., Mehta, A.O., Kim, S.B., Bachelot, T. and Goswami, C., 2016. Neratinib plus paclitaxel vs trastuzumab plus paclitaxel in previously untreated metastatic ERBB2-positive breast cancer: the NEfERT-T randomized clinical trial. JAMA oncology, 2(12), pp.1557-1564.

7. Györffy, B., Lanczky, A., Eklund, A.C., Denkert, C., Budczies, J., Li, Q. and Szallasi, Z., 2010. An online survival analysis tool to rapidly assess the effect of 22,277 genes on breast cancer prognosis using microarray data of 1,809 patients. Breast cancer research and treatment, 123(3), pp.725-731.

8. Zhou, K., Liu, Y., Zhao, Z., Wang, Y., Huang, L., Chai, R., Li, G. and Jiang, T., 2020. $\mathrm{C} 17$ orf37 mRNA expression is an independent prognostic factor for glioma and can predict chemosensitivity. Scientific Reports, 10(1), pp.1-11. 
Rank: 29

2 Probe ID: 10389

p-value: $3.76 \mathrm{E}-05$

$3 \quad \underline{\mathrm{t}}-5.66$

$4 \quad$ B: 2.416178

Gene: C17orf37

$5 \quad$ Gene name: open reading frame 37 on chromosome 17

Chart 1: C17orf37 is differentially expressed in brain metastatic breast cancer when comparing brain metastases to primary tumors of the breast.

The rank of global differential expression, the probe/transcript ID, the $p$-value with respect to differential expression transcriptome-wide, $\mathrm{t}$, a moderated $\mathrm{t}$-statistic, $\mathrm{B}$, the log-odds of differential expression between the groups compared, the gene and gene name are listed in this chart. 


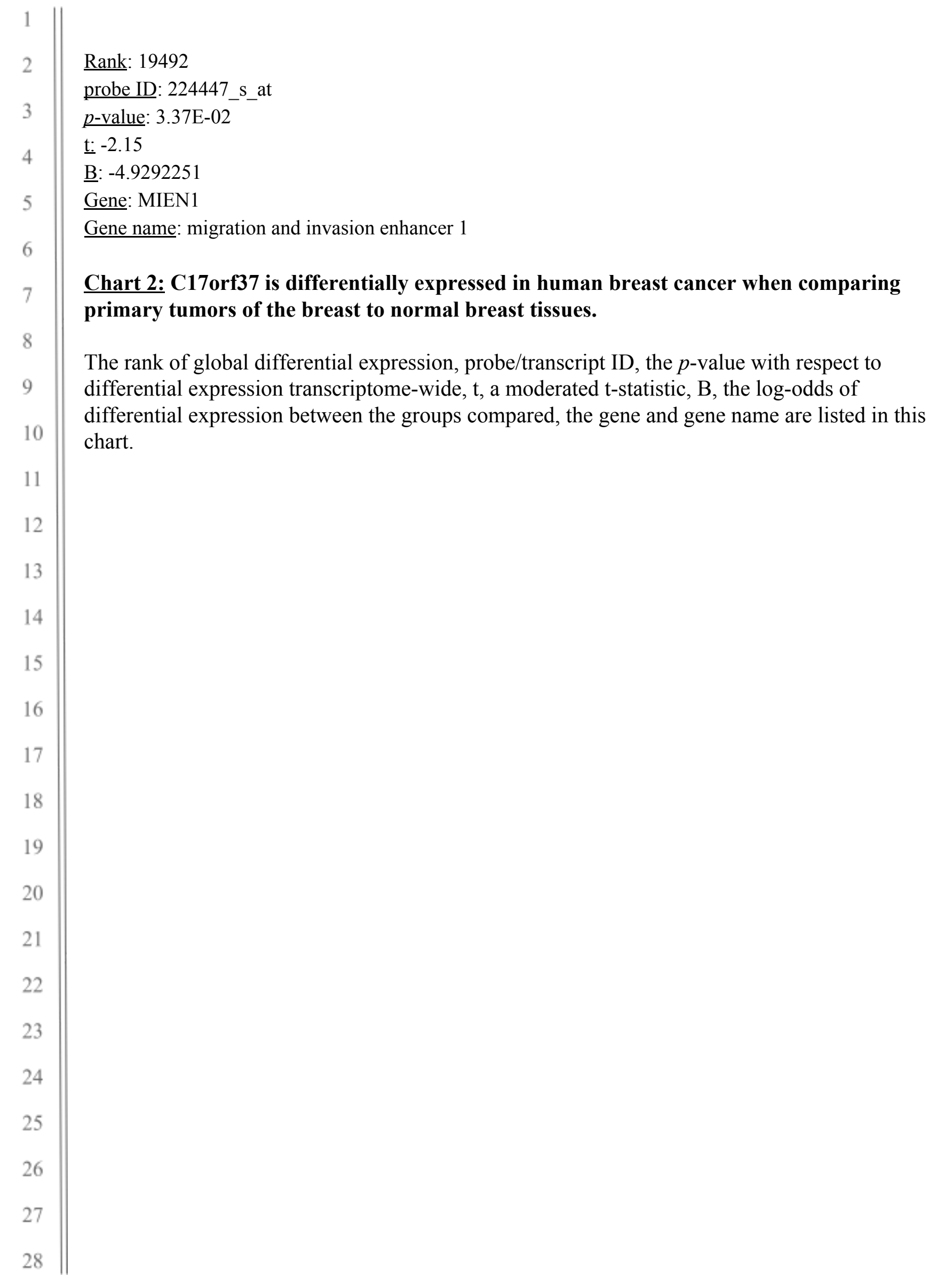




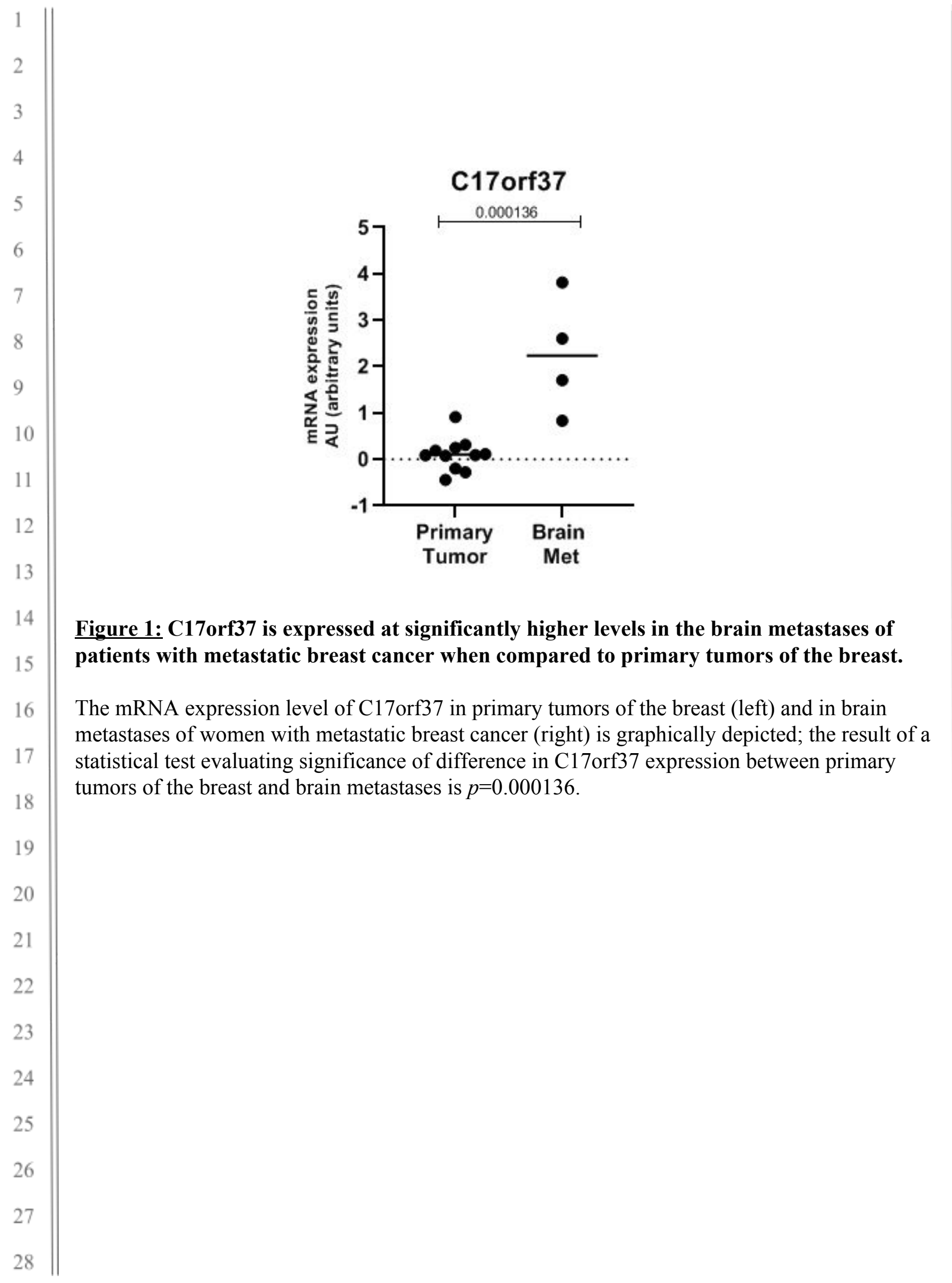

